\title{
Consanguinity in patients with mesial temporal lobe epilepsy due to hippocampal sclerosis in a Saudi population
}

Khalid S. Alqadi, MD, Saba A. Rammal, MD, Mosaab E. Alam, MD, Ashwaq M. Alshahrani, MD, Saleh S. Baeesa, MD, FRCSC, Husam R. Kayyali, MD, Fawzi A. Babtain, MD, Youssef A. Al-Said, MD, FRCPC.

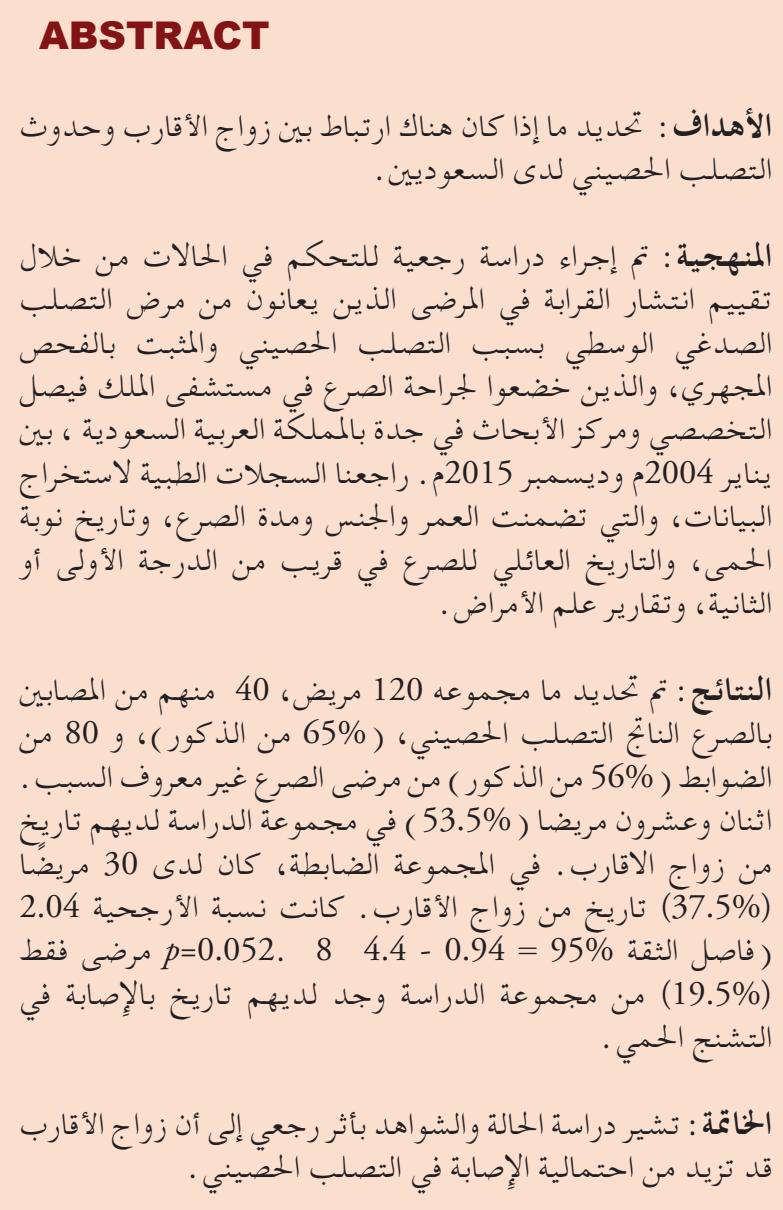

Objectives: To investigate if there is an association between consanguinity and hippocampal sclerosis (HS) in the Saudi population.

Methods: A retrospective case-control study was conducted by assessing the prevalence of consanguinity in patients with pathologically proven HS, who underwent epilepsy surgery at King Faisal Specialist Hospital and Research Center, Jeddah, Saudi Arabia, between January 2004 and December 2015. We reviewed the medical records to extract data, which included; age, gender, duration of epilepsy, history of febrile seizure, family history of epilepsy in a first or second-degree relative, and pathology reports.

Results: A total of 120 patients, out of which 40 patients (65\% male) having mesial temporal lobe epilepsy due to HS, and 80 controls (56\% male) with cryptogenic epilepsy, were identified. Twentytwo patients $(53.5 \%)$ in the HS group had a history of consanguinity. In the control group, 30 patients $(37.5 \%)$ had a history of consanguinity. The odds ratio was $2.04(95 \%$ confidence interval $=0.94-4.4$, $p=0.052$ ). A family history of epilepsy was found in $28 \%$ of the patients with HS and $32.5 \%$ cryptogenic epilepsy. Only 8 patients (19.5\%) with HS reported a history of febrile seizure.

Conclusions: Our retrospective case-control study suggests that consanguinity might increase the likelihood of developing HS.

\section{Neurosciences 2020; Vol. 25 (4): 276-280 doi: 10.17712/nsj.2020.4.20200055}

From the Department of Neurosciences (Alqadi, Alshahrani, Baeesa, Kayyali, Babtain, Al-Said), King Faisal Specialist Hospital, and Research Center, and from the Faculty of Medicine, King Abdulaziz University (Rammal, Alam, Baeesa), Jeddah, Kingdom of Saudi Arabia.

\section{Received 6th October 2019. Accepted 15th April 2020.}

Address correspondence and reprint request to: Dr. Khalid Alqadi, Department of Neurosciences, King Faisal Specialist Hospital \& Research Center, Jeddah, Kingdom of Saudi Arabia. E-mail:Dr.k.alqadi@gmail.com

ORCID ID: https://orcid.org/0000-0002-3695-3538
Consanguineous marriage, in clinical genetics, is defined as a union between couples related as second cousins or closer. ${ }^{1}$ Saudi Arabian culture has a higher consanguinity rate than other Arab, Asian, and Western communities. Consanguinity is not known to increase the risk of idiopathic or cryptogenic epilepsy, based on recent studies from Saudi Arabia and UAE. 2,3 
However, there is a strong evidence that the marriage of first cousins is one of the main reasons for the increased prevalence of autosomal recessive diseases. ${ }^{4}$

The association between consanguinity and epilepsy due to hippocampal sclerosis (HS), the most common pathology found in patients with intractable focal epilepsy, has not been adequately studied..$^{5}$ A familial type of mesial temporal lobe epilepsy (FMTLE) has been described with homogeneous and heterogeneous clinical manifestations. ${ }^{6-8}$ Moreover, magnetic resonance imaging (MRI) of the brain of asymptomatic firstdegree relatives of patients with confirmed FMTLE showed evidence of HS, which supports the genetic predisposition to FMTLE. Although, FMTLE is widely recognized as an autosomal dominant disease with incomplete penetrance, autosomal recessive or $\mathrm{X}$-linked forms have also been reported. Still, they have not been confirmed by extensive studies. ${ }^{10}$

In this study, we aimed to investigate the association between consanguinity and HS in a Saudi population. This study may help expand our knowledge of the underlying mechanisms of HS and may shed some light on a possible genetic substrate that contributes to the development of HS in this population.

Methods. This retrospective case-control study aimed to explore the prevalence of consanguinity in patients with pathologically proven HS, who underwent temporal lobectomy between January 2004 and December 2015, at King Faisal Specialist Hospital and Research Center, Jeddah, Saudi Arabia.

Patients with cryptogenic epilepsy were used as a control group. The case ratio between the control group and the study group was 1:2. The institutional review board approved the study (IRB\# 2015-60). Patients' consent was waived due to the retrospective nature of the study.

Medical records of the patients were reviewed to extract data, and patients with incomplete data were contacted, and verbal consent was obtained for the missing data. Data collected included their age, gender, the onset of epilepsy and the duration, history of febrile seizure (FS), family history of epilepsy in a first or seconddegree relative, neuroimaging findings, and pathology reports. We included all operated patients with messiah temporal lobe epilepsy (MTLE) due to pathologically-

Disclosure. Authors have no conflict of interests, and the work was not supported or funded by any drug company. confirmed HS. Patients with symptomatic MTLE were excluded due to pathologies other than HS.

In the control group, we included patients who have had focal and generalized cryptogenic epilepsy consecutively, while patients with symptomatic or idiopathic epilepsy were excluded.

Patients with cryptogenic epilepsy were chosen as the control group because we aimed to investigate the potential genetic background of HS, we wanted to compare patients with HS to a control group with epilepsy that is not known to be of genetic or structural etiology. This requirement does not apply to symptomatic epilepsy because of the structural etiology nor does it apply to idiopathic epilepsy due to its probable genetic cause.

On the other hand, cryptogenic epilepsy was chosen as a control group because it possesses the characteristics of epilepsy of unknown etiology.

Statistical analysis was performed using SPSS version 16.0 for Windows (SPSS Inc., Chicago, IL, USA). The ratio of the case-control was 1:2. The odds ratio

Table 1 - Chi-square and risk estimate.

\begin{tabular}{lcc}
\hline Patients with HS & Odds ratio- 95\% CI & $P$-value \\
\hline $\begin{array}{l}\text { Consanguinity 1st and 2nd } \\
\text { cousins }\end{array}$ & $2.037(0.943-4.40)$ & 0.052 \\
First cousin & $1.400(0.630-3.11)$ & 0.417 \\
Second cousin & $2.698(0.840-8.66)$ & 0.117 \\
\hline
\end{tabular}

HS: hippocampal sclerosis

(OR) with a confidence interval (CI) was used for risk estimation of consanguinity in the 2 groups, using the chi-squared test. Fisher's exact test was used to calculate the P-value (Table 1).

Results. A total of 40 patients with MTLE due to HS (65\% males) and 80 controls (56\% males) with cryptogenic epilepsy were identified (Table 2). Twenty-two patients (55\%) in the HS group had a history of consanguinity, with majority of the cases involving first cousins (68\%). In the control group, 30 patients $(37.5 \%)$ had a history of consanguinity, with $80 \%$ of the cases involving first cousins. The estimated chance of consanguinity occurrence in the HS group, in comparison with the control group, was statistically significant (OR, 2.04 [95\% CI = 0.94-4.4], $p=0.052$ ).

A family history of epilepsy (first and second degree) was found in $11(28 \%)$ patients with HS and $26(32 \%)$ 
Table 2 - Characteristics of 120 patients.

\begin{tabular}{lcc}
\hline Variables & $\begin{array}{c}\text { HS group } \\
(\mathbf{n}=\mathbf{4 0})\end{array}$ & $\begin{array}{c}\text { Control group } \\
(\mathbf{n}=80)\end{array}$ \\
\hline $\begin{array}{l}\text { Gender } \\
\text { Male }\end{array}$ & 26 & 45 \\
Female & 14 & 35 \\
Mean age at onset (range) & 11.5 years & 13 years \\
& $(1-36)$ & $(1-50)$ \\
Mean age at diagnosis (range) & 30 years & 31 years \\
& $(15-54)$ & $(9-68)$ \\
Parental consanguinity & $22(55.0)$ & $30(37.5)$ \\
$\quad$ First cousin & $15(68.0)$ & $24(80.0)$ \\
Second cousin & $7(32.0)$ & $6(20.0)$ \\
History of febrile seizure & $8(19.5)$ & $9(11.0)$ \\
The family history of epilepsy & $11(28.0)$ & $26(32.0)$ \\
Seizure freedom & $26(65.0)$ & $34(42.5)$ \\
\hline
\end{tabular}

HS: hippocampal sclerosis, "Seizure freedom after epilepsy surgery in HS group and seizure control in patients with cryptogenic epilepsy at the time of conducting the study

patients with cryptogenic epilepsy. Eight (19.5\%) patients in the HS group, and $9(11 \%)$ patients in the control group had a history of FS.

Successful surgical outcome was seen in $65 \%$ of the patients with HS.

Discussion. This retrospective case-control study was conducted at one of the epilepsy centers in Saudi Arabia, to determine if consanguinity is associated with HS in patients with TLE. The ratio of the number of individuals in the HS group to those in the control group in this study was $1: 2$. This study suggests that parental consanguinity might have a positive association with HS. A review on focal drug-resistant epilepsy by a Moroccan group found that mesial temporal lobe syndrome is associated with a high frequency of febrile convulsions and parental consanguinity. ${ }^{11}$ However, their cohort included only 6 patients with parental consanguinity. The association between consanguinity and epilepsy was also investigated by Babtain et $\mathrm{al}^{2}{ }^{2}$ who observed a lack of association between first-degree consanguinity and the risk of developing epilepsy, in the southern region of Saudi Arabia. However, in this study, second-degree consanguinity increased the risk of developing epilepsy. The social stigma of epilepsy possibly contributes to the observed lack of association between epilepsy and first-degree consanguinity. It seems that due to unfortunate social stigma experienced by patients with epilepsy, people with epilepsy avoid getting married to first-degree relatives, and they also think of avoiding the "transmit" of the disease to their children should they indulge.

Thus, a second-degree marriage is quite common, as an alternative to first-degree in Saudi Arabia and considered "ideal way" by many families to avoid having children with genetically inherited disease. It is well known that a family history of epilepsy is strongly associated with epilepsy, which was also noted in this study. Khan et $\mathrm{al}^{3}$ examined the association between parental consanguinity and family history with the risk of developing idiopathic epilepsy; 17\% of the observed epilepsy cases were associated with a history of parental consanguinity, but consanguinity was also observed in $31 \%$ of the control group, which consists of nonepileptic cohort extracted randomly from the same hospital record. On the other hand, a family history of epilepsy was observed in $81 \%$ of the epilepsy cases, while it was found in only $17 \%$ of the controls. It is worthy of note that this study considered only 76 patients. ${ }^{3}$ It is also important to note that a family history of epilepsy seems to have a strong association with the risk of developing epilepsy in the 2 studies mentioned above. This observation was validated in this current study. In the same Saudi population, a relationship between parental consanguinity and presumed familial type of multiple sclerosis was reported by Jumah et al. ${ }^{12}$ Such findings and the ones from this study may contribute to the field by enhancing existing knowledge regarding the risk of developing such neurological disorders without known inheritance patterns or described association with parental consanguinity.

Our results suggested that consanguineous marriage could be a direct or indirect contributing factor in focal epilepsy due to HS.

Gambardella et al, ${ }^{13}$ reported 8 patients from Italy with autosomal dominant FMTLE, but with incomplete penetrance, all patients had controlled seizures on antiepileptic drugs. In another study on FMTLE, a benign pattern was also observed in patients with and without HS; the prevalence of refractory epilepsy in FMTLE is less than that in typical surgical MTLE. Moreover, varying degrees of HS was observed in asymptomatic family members of patients with FMTLE, suggesting that the hippocampal abnormalities could be inherited. The degree of HS severity on MRI alone was not directly related to seizure severity and the development of refractory epilepsy, which is rather due to the complexity of MTLE pathogenesis and its underlying multifactorial etiologies. ${ }^{10}$ These findings are replicated in other studies where detecting HS in MRI 
was found in many patients with benign MTLE. ${ }^{14,15}$ However, a recent prospective study on patients with mild MTLE associated with the presence of HS leads to the progression of benign to refractory MTLE that requires surgical intervention. ${ }^{16}$ Genetic etiology has been demonstrated by several types of focal epilepsy in adult patients. The genetic basis of autosomal dominant partial epilepsy with auditory features was described by Winawer et al. ${ }^{17}$ The predisposing gene is localized in the $10 \mathrm{cM}$ regions on chromosome 10q24. Angelicheva et $\mathrm{al}^{18}$ examined the genetic basis of early-onset TLE syndrome in the Gypsy population. They identified an association between the syndrome and the loci $5 \mathrm{q} 31.3-\mathrm{q} 32$ and $10 \mathrm{p} 12$. These findings suggest that genetic susceptibility increases the likelihood of developing focal epilepsy. Our findings of linking consanguinity to HS, may emphasize the same notion. On the contrary, Striano et $\mathrm{al}^{19}$ reported clinical and genetic findings from 15 Italian families with FMTLE. The clinical and genetic findings were heterogeneous and could be linked to the studied loci or mutations.

We also observed an association between the history of FS and HS, found in $19.5 \%$ of the HS group and $11 \%$ of the control group. It is important to point out that sodium channel mutations lead to a homogenous phenotype of FS that is associated with HS. Colosimo et $\mathrm{al}^{20}$ and Scheffer et $\mathrm{al}^{21}$ recently demonstrated these mutations (SCN1A or SCN1B). Colosimo et $\mathrm{a}^{20}$ illustrated that out of 13 affected family members, 2 exhibited HS. Both cases showed focal complex or nocturnal secondary generalized tonic-clonic seizures. Scheffer et $\mathrm{al}^{21}$ performed SCN1B analysis on 402 individuals, only one of whom exhibited HS. This favors the hypothesis of a common genetic basis for TLE and FS, with or without HS.

In this study, we found that $65 \%$ of the patients who underwent anterior mesial temporal lobe resection had an excellent surgical outcome and became seizure-free after the surgery. ${ }^{22}$ The rate of seizure freedom is similar to what is reported in the literature for this type of epilepsy surgery. ${ }^{23,24}$

Study limitations. This study was conducted in only one tertiary epilepsy center. Hence, our sample size was relatively small. A larger sample size, with patients from multiple centers, may provide higher statistical credibility to the results.

In conclusion, this study demonstrates a potential association between consanguinity and HS, which is a contributing factor to this disorder. A more extensive national cohort study is needed to further confirm this association.

\section{References}

1. Bittles A. Consanguinity and its relevance to clinical genetics. Clin Genet 2001; 60: 89-98.

2. Babtain F, Al-Shahrani A, Assiri K, Al-Kahaili A, Bhatia H, Velmurugan $\mathrm{M}$ : Is parental consanguinity a risk factor for epilepsy? A cases-control study assessing epilepsy risks in Southern Saudi Arabia. Al-Azhar Assiut Medical Journal 2015; 13: 60-66.

3. Khan H, Mohamed A, Al-Sakini Z, Zulfiquar K, Sohail A, Shaikh RB, Al Sharbatti S, Mathew E: Consanguinity, family history, and risk of epilepsy: a case-control study. GulfMedical Journal 2012; 1: 32-36.

4. Stoltenberg C, Magnus P, Skrondal A, Lie RT: Consanguinity and recurrence risk of birth defects: a population-based study. Am J Med Genet 1999; 82: 423-428.

5. Falconer MA. Mesial temporal (Ammon's horn) sclerosis as a common cause of epilepsy. Aetiology, treatment, and prevention. Lancet 1974; 2: 767-770.

6. Berkovic SF, McIntosh A, Howell RA, Mitchell A, Sheffield LJ, Hopper JL: Familial temporal lobe epilepsy: a common disorder identified in twins. Ann Neurol 1996; 40: 227-235.

7. Cendes F, Lopes-Cendes I, Andermann E, Andermann F. Familial temporal lobe epilepsy: a clinically heterogeneous syndrome. Neurology 1998; 50: 554-557.

8. Wieser GH. ILAE Commission on Neurosurgery of Epilepsy, Mesial temporal lobe epilepsy with hippocampal sclerosis. Epilepsia 2004; 45: 695-714.

9. Kobayashi E, Li LM, Lopes-Cendes I, Cendes F: Magnetic resonance imaging evidence of hippocampal sclerosis in asymptomatic, first-degree relatives of patients with familialmesial temporal lobe epilepsy. Arch Neurol 2002; 59: 1891-1894.

10. Gambardella A, Labate A, Giallonardo A, Aguglia U. Familial mesial temporal lobe epilepsies: clinical and genetic features. Epilepsia 2009; 50: 55-57.

11. Souirti Z, Sghir A, Belfkih R, Messouak O: Focal drug-resistant epilepsy: progress in care and barriers, a Moroccan perspective. J Clin Neurosci 2016; 34: 276-280.

12. Al Jumah M, Kojan S, Al Khathaami A, Al Abdulkareami I, Al Blawi M, Jawhary A. Familial multiple sclerosis: does consanguinity have a role? Mult Scler 2011; 17: 487-489.

13. Gambardella A, Messina D, Le Piane E, Oliveri RL, Annesi G, Zappia M, Andermann E, Quattrone A, Aguglia U. Familial temporal lobe epilepsy: autosomal dominant inheritance in a large pedigree from Southern Italy. Epilepsy Research 2000; 38 : 127-132.

14. Labate A, Gambardella A, Andermann E, Aguglia U, Cendes F, Berkovic SF, Andermann F. Benign mesial temporal lobe epilepsy. Nature Reviews Neurology 2011; 7: 237.

15. Labate A, Ventura P, Gambardella A, Le Piane E, Colosimo E, Leggio U, Ambrosio R, Condino F, Messina D, Lanza P, Aguglia U. MRI evidence of mesial temporal sclerosis in sporadic "benign" temporal lobe epilepsy. Neurology 2006; 66: 562-565.

16. Labate A, Aguglia U, Tripepi G, Mumoli L, Ferlazzo E, Baggetta R, Quattrone A, Gambardella A. Long-term outcome of mild mesial temporal lobe epilepsy: a prospective longitudinal cohort study. Neurology 2016; 86: 1904-1910. 
17. Winawer MR, Martinelli Boneschi F, Barker-Cummings C, Lee JH, Liu J, Mekios C, Gilliam TC, Pedley TA, Hauser WA, Ottman R: Four new families with autosomal dominant partial epilepsy with auditory features: clinical description and linkage to chromosome 10q24. Epilepsia 2002; 43: 60-67.

18. Angelicheva D, Tournev I, Guergueltcheva V, Mihaylova V, Azmanov DN, Morar B, et al. Partial epilepsy syndrome in a Gypsy family linked to 5q31.3-q32. Epilepsia 2009; 50: 1679-1688.

19. Striano P, Gambardella A, Coppola A, Di Bonaventura C, Bovo G, Diani E, Boaretto F, Egeo G, Ciampa C, Labate A, Testoni S. Familial mesial temporal lobe epilepsy (FMTLE). Journal of Neurology 2008; 255: 16-23.

20. Colosimo E, Gambardella A, Mantegazza M, Labate A, Rusconi R, Schiavon E, et al. Electroclinical features of a family with simple febrile seizures and temporal lobe epilepsy associated with SCN1A loss-of-function mutation. Epilepsia 2007; 48: 1691-1696.
21. Scheffer IE, Harkin LA, Grinton BE, Dibbens LM, Turner SJ, Zielinski MA, et al. Temporal lobe epilepsy and GEFS+ phenotypes associated with SCN1B mutations. Brain 2007; 130: 100-109.

22. Engel J. Surgical Treatment of the Epilepsies. New York (NY): Lippincott Williams \& Wilkins; 1993.

23. McIntosh AM, Wilson, SJ, Berkovic SF. Seizure outcome after temporal lobectomy: Current research practice and findings. Epilepsia 2001; 42: 1288-1307.

24. Jeha LE, Najm IM, Bingaman WE, Khandwala F, WiddessWalsh P, Morris HH, Dinner DS, Nair D, Foldvary-Schaeffer N, Prayson RA, Comair Y. Predictors of outcome after temporal lobectomy for the treatment of intractable epilepsy. Neurology 2006; 66: 1938-1940.

\section{Withdrawal policy}

By submission, the author grants the journal right of first publication. Therefore, the journal discourages unethical withdrawal of manuscripts from the publication process after peer review. The corresponding author should send a formal request signed by all co-authors stating the reason for withdrawing the manuscript. Withdrawal of a manuscript is only considered valid when the editor accepts, or approves the reason to withdraw the manuscript from publication. Subsequently, the author must receive a confirmation from the editorial office. Only at that stage, are the authors free to submit the manuscript elsewhere.

No response from the authors to all journal communication after review and acceptance is also considered unethical withdrawal. Withdrawn manuscripts noted to have already been submitted or published in another journal will be subjected to sanctions in accordance with the journal policy. The journal will take disciplinary measures for unacceptable withdrawal of manuscripts. An embargo of 5 years will be enforced for the author and their co-authors, and their institute will be notified of this action. 